\title{
Influencia del retraso en el procesado de las aceitunas tras la recolección, en parámetros físico-químicos y nutricionales del aceite de oliva de la variedad Racimilla
}

\author{
Por M. Benito, R. Oria and A. C. Sánchez-Gimeno* \\ Tecnología de los Alimentos. Facultad de Veterinaria. \\ Universidad de Zaragoza- Miguel Servet, 177 - 50013 Zaragoza (Spain) \\ ( ${ }^{\star}$ Autor para la correspondencia: anacris@ unizar.es)
}

\section{RESUMEN}

Influencia del retraso en el procesado de las aceitunas tras la recolección, en parámetros físico-químicos y nutricionales del aceite de oliva de la variedad Racimilla.

El objetivo de este trabajo fue estudiar cómo afecta el tiempo que transcurre entre la recolección y procesado en los parámetros físico-químicos y nutricionales del aceite de oliva (Olea europaea L.) de la variedad aragonesa Racimilla. Los parámetros físico-químicos (acidez, índice de peróxidos, coeficientes de extinción y contenido en pigmentos) se modificaron visiblemente. Los parámetros nutricionales como el perfil de ácidos grasos, contenido en fenoles totales y vitamina $\mathrm{E}$ sufrieron también un cambio al retrasar el procesado. Tanto las modificaciones en los parámetros físico-químicos como en los nutricionales fueron negativas desde el punto de vista de la calidad del aceite. A la vista de los resultados, el retraso en el procesado tras la recolección es ineficiente para preservar la calidad físico-química y nutricional del aceite de oliva de la variedad Racimilla.

PALABRAS CLAVE: Aceite de oliva - Aceituna - Almacenamiento - Calidad - Degradación - Procesado.

\section{SUMMARY}

Effect of the delay in olive processing after harvesting on some physicochemical and nutritional parameters of olive oil from the Racimilla variety.

The aim of this work was to study how the delay time between harvesting and processing affects the physicochemical and nutritional parameters of Racimilla olive (Olea europaea L.) oil. The physicochemical parameters (titratable acidity, peroxide value, coefficients of specific extinction and pigments content) changed remarkably. The nutritional parameters such as fatty acids profile, total phenols and vitamin E also suffered a change with the delay in processing. Physicochemical and nutritional parameter modifications were detrimental to olive oil quality. In conclusion, a delay in processing after harvesting is not efficient to preserve the physicochemical and nutritional quality of the olive oil from the Racimilla variety.

KEY-WORDS: Degradation - Olive - Olive oil Processing - Quality - Storage.

\section{INTRODUCCIÓN}

El aceite de oliva es el obtenido a partir de la aceituna exclusivamente por procedimientos mecá- nicos u otros medios físicos, en condiciones especialmente térmicas que no produzcan la alteración del aceite, que no haya tenido más tratamiento que el lavado, la decantación, la centrifugación y el filtrado (Aparicio y Harwood, 2003). En definitiva, el aceite de oliva es en estricto el zumo de la aceituna, poseedor de una gran calidad nutritiva, organoléptica y comercial y verdadero protagonista de la dieta mediterránea. Desde un punto de vista nutricional el aceite de oliva es un alimento de gran valor, derivado de su elevado contenido en ácidos grasos monoinsaturados (ácido oleico) y en compuestos antioxidantes como los fenoles y la vitamina $E$.

La calidad del aceite de oliva depende de muchos factores: la variedad de aceituna, el clima, las condiciones edáficas, método de recolección, grado de madurez de la aceituna, proceso de extracción y factores agronómicos fundamentalmente (Salvador et al., 2001; Beltrán et al., 2005). Uno de los factores más importantes responsables de la pérdida de calidad del aceite de oliva es el aplazamiento del procesado de las aceitunas tras la recolección. En estas circunstancias las aceitunas permanecen amontonadas a la espera de su molienda, desarrollándose muchos procesos de deterioro en poco tiempo por calentamiento, fermentaciones, hidrólisis y desarrollo de microorganismos (García y Yousfi, 2006; Uceda et al., 2006). Este deterioro en el aceite obtenido a partir de estas aceitunas se manifiesta tanto en los parámetros físico-químicos como en los organolépticos. Para reducirlo se aplican diversas tecnologías postcosecha, aunque las almazaras que pretenden obtener aceites de oliva de elevada calidad procesan inmediatamente tras la cosecha, en ocasiones en plazos inferiores a tres horas entre ambas. En todo caso deben procesarse a ser posible en un periodo de tiempo inferior a 24 horas desde su recogida, lo que exige aumentar la capacidad de molturación (Toro et al., 2002). Sin embargo, el aumento de la maquinaria requerida para ello supone elevadísimas inversiones económicas. Si se tiene en cuenta que la obtención de aceite de oliva tiene un carácter estacional, y que dicha maquinaria es utilizada menos de la mitad del año y sólo para este fin, resulta muy caro optar por esta solución. Existen algunas tecno- 
logías postcosecha como la conservación en refrigeración de las aceitunas para evitar el deterioro de los aceites en las aceitunas cuyo procesado se retrasa (García et al., 1996a).

La calidad de los aceites de oliva viene definida por el Reglamento CE 2568/ 1991 y posteriores modificaciones (Rgto CE 1989/ 2003 y 640/ 2008) relativo a las características de los aceites de oliva y de los aceites de orujo de oliva y sobre sus métodos de análisis. Estas normas establecen la clasificación comercial de los aceites en función de parámetros físico-químicos (acidez libre, índice de peróxidos, coeficientes de extinción específica, etc.) y sensoriales en aceite de oliva virgen extra (sin defectos y con una acidez máxima de $0.8^{\circ}$ ), aceite de oliva virgen (con un máximo de 3.5 en defectos y acidez menor o igual $\mathrm{a}^{\circ}$ ) y aceite de oliva lampante (con más de 3.5 puntos en defectos y acidez superior a $2^{\circ}$ ) que no puede ser destinado al consumo humano y debe ser refinado para su consumo.

Existen algunos estudios previos sobre la influencia del almacenamiento de las aceitunas en la alteración de algunos parámetros físico-químicos del aceite aunque, no se han realizado en las mismas condiciones que las de este trabajo, ni en las mismas variedades de aceitunas, y se han limitado al estudio del efecto sobre los parámetros de calidad exigidos por la legislación, sin entrar a determinar cómo afecta el almacenamiento a otros parámetros importantes como los nutricionales (fenoles, vitamina $\mathrm{E}$, etc). Camera et al., (1978) investigaron en variedades de aceitunas italianas el efecto del almacenamiento en la fracción esterólica del aceite, así como en la acidez. La acidez y cantidad total de esteroles aumentaron. García et al., (1996a) estudiaron en las variedades Blanqueta y Villalonga conservadas a $5^{\circ} \mathrm{C}$ y a temperatura ambiente el efecto del almacenamiento en la acidez, índice de peróxidos y coeficientes de extinción que se incrementaron en la conservación a temperatura ambiente, aunque el deterioro se ralentizó en refrigeración.

El objetivo de este trabajo fue investigar el efecto de un procesado tardío de las aceitunas tras la recolección (práctica habitual en algunas almazaras tradicionales) en diversos parámetros de calidad (físico-químicos y nutricionales) del aceite de oliva obtenido a partir de una variedad aragonesa, la Racimilla, variedad de notable importancia en algunas comarcas de la provincia de Zaragoza, donde la variedad Empeltre es minoritaria (aún cuando esta última variedad es la mayoritaria en Aragón en especial en la provincia de Teruel).

\section{MATERIALES Y MÉTODOS}

\subsection{Materia prima}

Las aceitunas de la variedad Racimilla (Olea Europea Racemosa) (Priego, 1930) fueron recolectadas en diciembre de 2007 en olivares de secano de la comarca de Valdejalón (Zaragoza) de forma manual (mediante la técnica de ordeño). Tras su transporte al laboratorio se procedió a la determinación del índice de madurez de las aceitunas siguiendo el método de la estación de Olivicultura y Elaiotecnia de Jaén (Hermoso et al., 1991) y a la extracción del aceite de la muestra control. El resto de aceitunas se almacenaron en un recinto a temperatura ambiente $\left(15 \pm 2^{\circ} \mathrm{C}\right)$ y $75 \pm 3 \%$ humedad relativa $(\mathrm{HR})$ en cajas de plástico perforadas de 20 $\mathrm{Kg}$ (conteniendo $20 \mathrm{~kg}$ de aceitunas) durante 12, 18 y 20 días antes de proceder a su procesado para simular el almacenamiento que tiene lugar en las almazaras en algunas ocasiones.

\subsection{Extracción del aceite y cálculo del rendimiento}

El aceite se obtuvo utilizando un equipo Abencor (MC2, Sevilla, España) (Martínez- Suárez et al., 1975). Las olivas se trituraron mediante un molino de martillos accionado por un motor de $2 \mathrm{CV}$ a $3000 \mathrm{rpm}$. La pasta resultante se sometió a batido en una termobatidora a $26{ }^{\circ} \mathrm{C}$ durante 30 minutos y posteriormente se vertió dentro de la centrífuga vertical de tipo cesta que gira a 3500 rpm y se accionó durante 1 minuto. La centrifugación se repitió de nuevo. Se recogió el mosto oleoso, por el orificio inferior en una probeta graduada. Tras reposo de al menos 30 minutos se efectuó la lectura del volumen de aceite y se calculó el rendimiento de la operación con la siguiente fórmula:

$$
\mathrm{R}=\frac{\mathrm{V} \times \mathrm{d}}{\mathrm{P}} \times 100
$$

Siendo, R: Rendimiento de aceite en peso fresco (\%); V: Volumen de aceite obtenido $(\mathrm{mL})$; d: densidad del aceite considerando una temperatura de $20{ }^{\circ} \mathrm{C}(0.915 \mathrm{~g} / \mathrm{ml})$; P: peso de pasta batida.

El aceite se recuperó y posteriormente se filtró y conservó en botellas ámbar de cristal a $15^{\circ} \mathrm{C}$ en condiciones óptimas para realizar posteriormente los análisis correspondientes.

\subsection{Determinación de la acidez}

Para la determinación de la acidez se empleó el método oficial del Anexo II del Reglamento de la CEE 2568/91 (1991). La acidez se expresó en porcentaje de ácido oleico.

\section{4. Índice de Peróxidos}

Para su determinación se siguió el procedimiento descrito en el Anexo III del Reglamento de la CEE 2568/91. El resultado se expresó en miliequivalentes de oxígeno activo por $\mathrm{Kg}$ de muestra.

\subsection{Ensayo espectrofotométrico en el ultravioleta}

Para la determinación de los coeficientes de extinción se empleó el método oficial del Anexo IX del Reglamento de la CEE 2568/91 (1991). 


\subsection{Determinación del contenido en pigmentos}

Para la determinación de clorofilas y carotenoides en los aceites se realizaron por espectrofotometría espectros de transmisión de los aceites en un rango de longitudes de onda de 380 a $780 \mathrm{~nm}$ en intervalos de $2 \mathrm{~nm}$ (usando cubetas de vidrio de $1 \mathrm{~cm}$ y hexano como referencia). Para el cálculo del contenido en pigmentos se utilizó el método descrito por Mínguez- Mosquera et al., (1991) basado en la estimación cuantitativa de la fracción clorofílica por medida de la absorbancia del aceite a $670 \mathrm{~nm}$ y de la fracción carotenoides a $470 \mathrm{~nm}$ utilizando los coeficientes de absorción molar apropiados. Se utilizan para ello las siguientes fórmulas:

$$
\begin{gathered}
\text { Clorofilas }=\left(A_{670} \times 10^{6}\right) /(613 \times 100 \times \text { densidad }) \\
\text { Carotenoides }=\left(A_{470} \times 10^{6}\right) /(2000 \times 100 \times \text { densidad })
\end{gathered}
$$

Siendo 613 y 2000 los coeficientes de absorción molar de la feofitina a y de la luteína respectivamente. Los resultados se expresan en $\mathrm{mg}$ de feofitina a/ Kg de aceite en el caso de las clorofilas y $\mathrm{mg}$ luteína/ Kg aceite en el caso de los carotenoides.

\subsection{Determinación de los ácidos grasos por CG}

Los ácidos grasos totales fueron transmetilados de acuerdo con el método de Frega y Bocci (2001). Transcurridos 10 minutos, se inyectó la muestra $(0,4 \mu \mathrm{l})$ en un cromatógrafo de gases (Hewlett-Packard $5890 \mathrm{CG}$ ) equipado con un inyector split-splitless y un detector de ionización de llama. Se utilizó una columna DB-225 de $30 \mathrm{~m}$ de longitud y 0,25 $\mathrm{mm}$ de diámetro interno con un espesor de película de $0,15 \mu \mathrm{m}$ (J\&W Scientific, Agilent). El programa de temperaturas utilizado para la cromatografía fue: 1 min a $190^{\circ} \mathrm{C}$, una rampa de calentamiento hasta $210^{\circ} \mathrm{C}$ a $4{ }^{\circ} \mathrm{C} / \mathrm{min}$, una isoterma de $5 \mathrm{~min}$ a $210^{\circ} \mathrm{C}$, una rampa de calentamiento hasta $215^{\circ} \mathrm{C}$ a $3{ }^{\circ} \mathrm{C} / \mathrm{min}$ y, finalmente, una isoterma de $18 \mathrm{~min}$ a esta última temperatura.

Se realizó la identificación de los ácidos grasos existentes en la muestra metilada por comparación de los tiempos de retención presentes en el cromatograma obtenido con los correspondientes a los patrones de diversos ácidos grasos.

\subsection{Determinación del contenido en $\alpha$-tocoferol}

Se disolvió un gramo de muestra en $10 \mathrm{ml}$ de $\mathrm{n}$ hexano. La solución resultante se filtró a través de un filtro de nylon de 0,45 $\mu \mathrm{m}$. Se rellenó un vial de unos $2 \mathrm{ml}$ de capacidad provisto de tapón con septum. La muestra se inyectó en un cromatógrafo líquido de alta resolución Hewlett Packard (AgilentSerie 1100) compuesto de un desgasificador (G1322 A, Serie 1100), una bomba cuaternaria (G1311 A, Serie 1100), un inyector automático (G1313 A, Serie 1100) y un detector de matriz de fotodiodos (DAD) (G1315 B, Serie 1100). Se empleó una columna Zorbax $\mathrm{SB}^{-\mathrm{C}_{18}}$ de fase reversa (Agilent Technologies). El volumen de inyección fue

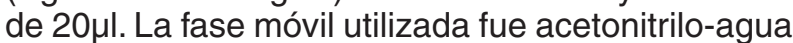
$(98: 2 \mathrm{v} / \mathrm{v})$ en condiciones isocráticas y el flujo de la fase móvil fue de $1 \mathrm{ml} / \mathrm{min}$. Los cromatogramas se registraron a $295 \mathrm{~nm}$. Se construyó la recta de calibrado a partir del estándar y se utilizó para la cuantificación. Los resultados fueron expresados en $\mathrm{mg}$ de $\alpha$-tocoferol por $\mathrm{Kg}$ de aceite.

\subsection{Determinación del contenido en fenoles totales}

Para la preparación de la muestra se adaptó el método descrito por Favati et al., (1994). Los fenoles fueron extraídos mediante extracción en fase sólida (SPE). Para ello se utilizaron columnas Isolute $\mathrm{C} 18$ que se equilibraron con metanol y hexano. Los fenoles se eluyeron con metanol y el eluato recogido $(10 \mathrm{ml})$ se evaporó hasta sequedad a $40{ }^{\circ} \mathrm{C}$ en el rotavapor redisolviéndose posteriormente en $5 \mathrm{ml}$.

Determinación colorimétrica de los fenoles totales con el reactivo de Folin-Ciocalteau

Se realizó una curva patrón de ácido gálico en metanol en un rango de concentración de 0,04-0,7 $\mathrm{mg} / \mathrm{ml} .1 \mathrm{ml}$ del extracto obtenido mediante SPE se depositó en un matraz de $50 \mathrm{ml}$. Se le añadieron a muestra y patrones $6 \mathrm{ml}$ de metanol, $2,5 \mathrm{ml}$ del reactivo de Folin-Ciocalteau y $5 \mathrm{ml}$ de carbonato sódico $(7,5 \%)$, alcanzando el volumen final con agua destilada. Las muestras se dejaron reposar toda la noche en oscuridad y se realizó el análisis espectrofotométrico a $765 \mathrm{~nm}$. El contenido en fenoles totales se expresó en $\mathrm{mg}$ de ácido gálico por $\mathrm{Kg}$ de aceite.

\subsection{Tratamiento estadístico}

Los resultados obtenidos de tres réplicas de las muestras fueron sometidos a un análisis estadístico utilizando el programa Graph Pad Prisma 5.0. Las diferencias significativas entre muestras fueron determinadas mediante el análisis de varianza y aplicación del test de Bonferroni con un nivel de significación del 95\% $(p<0.05)$.

\section{RESULTADOS Y DISCUSION}

El índice de madurez de las aceitunas control, calculado teniendo en cuenta la pigmentación del mesocarpio y epicarpio fue de $4.6 \pm 0.3$. Por tanto las aceitunas presentaban el epicarpio negro, con algo de color en el mesocarpio. Dicho índice se modificó muy ligeramente en las aceitunas sometidas a diferentes tiempos de almacenamiento y sólo tras los 10 primeros días de almacenamiento, permaneciendo constante en los posteriores (Tabla 1). 
Tabla 1

Rendimiento en aceite, índice de madurez y parámetros físico-químicos en aceite de oliva de la variedad Racimilla*

\begin{tabular}{lcccc}
\hline \multicolumn{1}{c}{ Parámetro } & Control & 12 días & 18 días & 20 días \\
\hline Rendimiento $(\%)$ & $28.36 \pm 1.30 \mathrm{a}$ & $31.23 \pm 0.90 \mathrm{ab}$ & $32.72 \pm 1.70 \mathrm{~b}$ & $39.04 \pm 2.10 \mathrm{c}$ \\
Índice de madurez & $4.6 \pm 0.3 \mathrm{a}$ & $5.7 \pm 0.4 \mathrm{~b}$ & $5.6 \pm 0.6 \mathrm{~b}$ & $5.8 \pm 0.5 \mathrm{~b}$ \\
Acidez $(\%$ ácido oleico) & $0.18 \pm 0.02 \mathrm{a}$ & $0.20 \pm 0.00 \mathrm{a}$ & $0.24 \pm 0.02 \mathrm{a}$ & $0.45 \pm 0.00 \mathrm{~b}$ \\
Índice de peróxidos $\left(\mathrm{meq} \mathrm{O}_{2} / \mathrm{kg}\right)$ & $2.66 \pm 0.01 \mathrm{a}$ & $6.42 \pm 0.21 \mathrm{~b}$ & $9.85 \pm 0.18 \mathrm{c}$ & $10.08 \pm 0.38 \mathrm{~cd}$ \\
$\mathrm{~K}_{270}$ & $0.10 \pm 0.00 \mathrm{a}$ & $0.11 \pm 0.00 \mathrm{~b}$ & $0.12 \pm 0.00 \mathrm{c}$ & $0.12 \pm 0.00 \mathrm{~cd}$ \\
$\mathrm{~K}_{232}$ & $1.69 \pm 0.01 \mathrm{a}$ & $1.92 \pm 0.01 \mathrm{~b}$ & $2.80 \pm 0.02 \mathrm{c}$ & $2.63 \pm 0.06 \mathrm{~cd}$ \\
\hline
\end{tabular}

* El aceite de oliva de la variedad Racimilla es extraído tras la recolección (control) y tras 12,18 y 20 días de almacenamiento (valor medio \pm desviación estándar, $n=3$ ). Distintas letras en la misma línea representan diferencias significativas.

Discriminación de medias con test de Bonferroni con un nivel de significación del 95\% $(p<0.05)$.

La Tabla 1 muestra los resultados experimentales del rendimiento de la extracción y del análisis de los diferentes parámetros físico-químicos exigidos por la legislación, en los aceites obtenidos inmediatamente tras la recolección (control) y tras un tiempo de almacenamiento (12, 18 y 20 días). El rendimiento de la extracción de aceite fue muy elevado en esta variedad (28.36\%). Dicho valor aumentó tras el almacenamiento, probablemente por la pérdida de agua de las aceitunas por evaporación. Los valores de acidez e índice de peróxidos aumentaron al aumentar el tiempo de almacenamiento como ha sido descrito por García et al., (1996a) en aceitunas de las variedades Blanqueta y Villalonga y por Camera et al., (1978) en variedades italianas. El aumento de los valores de acidez revela el desarrollo de reacciones de hidrólisis de los triglicéridos durante el almacenamiento con la consiguiente liberación de ácidos grasos cuantificados como ácido oleico. El aumento del índice de peróxidos supone el comienzo de reacciones de oxidación del aceite. Los valores de $\mathrm{K}_{270}$ y $\mathrm{K}_{232}$ siguieron la misma tendencia que los parámetros anteriores en los aceites obtenidos aumentando tras el almacenamiento de las aceitunas por comparación con el control hasta alcanzar un valor máximo tras 18 días de almacenamiento coincidiendo con García et al., (1996a) que describieron un incremento en estos parámetros. En todo caso, a pesar de la degradación, los aceites obtenidos de las aceitunas almacenadas se mantuvieron en general dentro de los límites establecidos por la legislación para estos parámetros (acidez, índice de peróxidos y $\mathrm{K}_{270}$ ) (Rgto 2568/ 1991 y modificaciones) para la clasificación comercial de los aceites en la categoría de aceite de oliva virgen extra. Sólo en el caso del pa- rámetro $\mathrm{K}_{232}$ se superaron los valores reglamentados para las categorías de aceite de oliva virgen extra y aceite de oliva virgen para los aceites obtenidos tras 18 y 20 días de almacenamiento que corresponderían a aceites de oliva lampantes.

Los resultados de la determinación de pigmentos en los aceites de oliva se muestran en la Tabla 2. Los aceites objeto de estudio fueron más ricos en pigmentos carotenoides que clorofílicos. Tanto el contenido en carotenoides como en clorofilas descendió al retrasar el procesado hasta alcanzar un mínimo a los 18 días de retraso en el procesado, no existiendo apenas diferencia en el contenido frente a los aceites procesados a los 20 días. Mientras el fruto está en el árbol y se desarrolla la maduración va disminuyendo progresivamente el contenido en clorofilas a la vez que aumenta el de carotenoides. Sin embargo al final del proceso de la maduración y tras la recolección y almacenamiento ambos contenidos disminuyen con la senescencia del fruto.

Además de los parámetros físico-químicos establecidos por la legislación se analizaron compuestos nutricionales como los perfiles de ácidos grasos, contenido en fenoles totales y en tocoferol. Por lo que respecta a los ácidos grasos, se muestra en la Tabla 3 el contenido en los principales ácidos grasos (palmítico, esteárico, oleico y linoleico) para las distintas muestras. Como puede observarse, los valores en los dos primeros apenas se modificaron con el almacenamiento, sin embargo, el contenido en ácido oleico disminuyó de forma significativa en los aceites que se obtuvieron a partir de aceitunas almacenadas a la vez que aumentó el contenido en ácido linoleico. Similares resultados fueron encontrados en aceites de oliva de variedades tunecinas en el curso de la maduración (Baccouri et al., 2008).

Tabla 2

\section{Contenido en carotenoides (mg luteína/ $\mathrm{Kg}$ aceite) y clorofilas (mg de feofitina a/ $\mathrm{Kg}$ de aceite)} en aceite de oliva de la variedad Racimilla*

\begin{tabular}{lcccc}
\hline Pigmento & Control & 12 días & 18 días & 20 días \\
\hline Carotenoides & $9.54 \pm 0.03^{\mathrm{a}}$ & $6.34 \pm 0.01 \mathrm{~b}$ & $5.26 \pm 0.00 \mathrm{c}$ & $5.39 \pm 0.01 \mathrm{~cd}$ \\
Clorofilas & $1.15 \pm 0.00 \mathrm{a}$ & $0.95 \pm 0.01 \mathrm{~b}$ & $0.77 \pm 0.05 \mathrm{c}$ & $0.79 \pm 0.00 \mathrm{~cd}$ \\
\hline
\end{tabular}

* EL aceite de oliva de la variedad racimilla es extraído tras la recolección (control) y tras 12,18 y 20 días de almacenamiento (valor medio \pm desviación estándar, $n=3$ ). Distintas letras en la misma línea representan diferencias significativas. Discriminación de medias con test de Bonferroni con un nivel de significación del 95\% $(p<0.05)$. 
Tabla 3

Contenido en los principales ácidos grasos (\%) en aceite de oliva de la variedad Racimilla*

\begin{tabular}{lcccc}
\hline Ácidos grasos & Control & 12 días & 18 días & 20 días \\
\hline Palmítico (\%) & $9.74 \pm 0.01 \mathrm{a}$ & $9.86 \pm 0.04 \mathrm{~b}$ & $9.90 \pm 0.01 \mathrm{bc}$ & $9.92 \pm 0.02 \mathrm{bcd}$ \\
Esteárico (\%) & $2.36 \pm 0.01 \mathrm{a}$ & $2.37 \pm 0.00 \mathrm{a}$ & $2.46 \pm 0.01 \mathrm{~b}$ & $2.49 \pm 0.00 \mathrm{bc}$ \\
Oleico (\%) & $77.20 \pm 0.00 \mathrm{a}$ & $76.15 \pm 0.11 \mathrm{~b}$ & $75.74 \pm 0.07 \mathrm{c}$ & $75.68 \pm 0.00 \mathrm{~cd}$ \\
Linoleico (\%) & $7.89 \pm 0.01 \mathrm{a}$ & $8.81 \pm 0.03 \mathrm{~b}$ & $9.13 \pm 0.02 \mathrm{c}$ & $9.19 \pm 0.02 \mathrm{~cd}$ \\
\hline
\end{tabular}

* El aceite de oliva de la variedad Racimilla es extraído tras la recolección (control) y tras 12, 18 y 20 días de almacenamiento (valor medio \pm desviación estándar, $n=3$ ). Distintas letras en la misma línea representan diferencias significativas.

Discriminación de medias con test de Bonferroni con un nivel de significación del 95\% $(p<0.05)$.

La Figura 1 muestra la evolución del contenido en alfa- tocoferol en los aceites. Como se puede comprobar, el almacenamiento de las aceitunas produce una degradación de este compuesto antioxidante del aceite. La disminución de este compuesto tiene también lugar según avanza la maduración de las aceitunas (García et al., 1996b). La Figura 2 refleja la evolución del contenido en fenoles totales del aceite en las distintas muestras. Tan sólo en 12 días de almacenamiento el contenido se redujo a la tercera parte aproximadamente. También durante la maduración, el contenido en fenoles totales, tras un máximo, comienza a decrecer (Montedoro et al., 1978). Ambos compuestos son los responsables de la estabilidad oxidativa del aceite y de su vida útil (Mateos et al., 2003).

\section{CONCLUSIONES}

Los resultados mostrados confirman que las condiciones de procesado retrasado de las aceitunas son ineficientes para preservar los parámetros de calidad físico-química y nutricional de los aceites obtenidos.

\section{AGRADECIMIENTOS}

Este trabajo ha sido realizado gracias a la concesión de una Ayuda para la adquisición de Infraestructura (Abencor) de la Universidad de Zaragoza y Go-

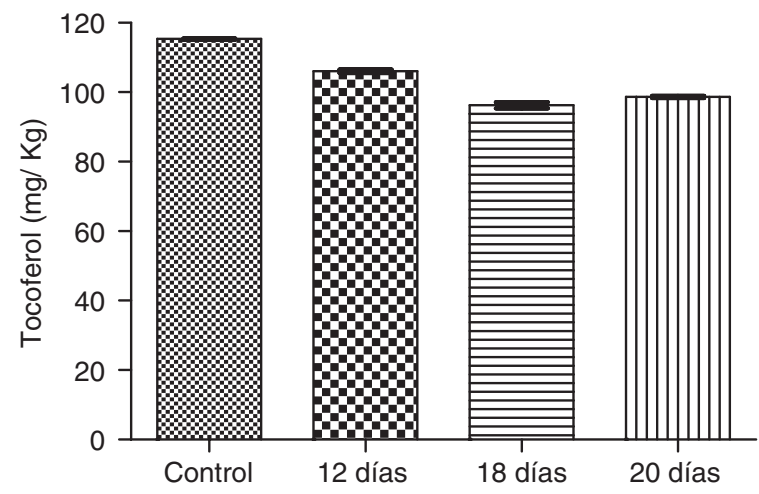

Figura 1

Contenido en alfa- tocoferol $(\mathrm{mg} / \mathrm{Kg}$ ) en aceite de oliva de la variedad Racimilla extraído tras la recolección (control) y tras 12,18 y 20 días de almacenamiento (valor medio \pm desviación estándar, $\mathrm{n}=3$ ). bierno de Aragón a Ana Cristina Sánchez Gimeno y a una beca predoctoral concedida por la Diputación General de Aragón a Marta Benito Velasco.

\section{REFERENCIAS}

Aparicio R, Harwood J. 2003. Manual del aceite de oliva. AMV Ediciones. Mundiprensa.

Baccouri O, Guerfel M, Baccouri B, Cerretani L, Bendini A, Lercker G, Zarrouk M, Ben Miled D D. 2008. Chemical composition and oxidative stability of Tunisian monovarietal virgen olive oil with regard to fruit ripening. Food Chemistry 109, 743- 754.

Beltrán G, Aguilera MP, Del Río C, Sánchez S, Martínez M. 2005. Influence of fruit ripening on the natural antioxidant content of Hojiblanca virgen olive oils. Food Chemistry 89, 207- 215.

Camera L, Angerosa F, Cucurachi A. 1978. Influenza dello stoccaggio delle olive sui costituenti della frazione sterolica dell' olio. Rivista Italiana delle Sostanze Grasse. 15, 107- 112.

Favati F, Caporale G, Bertuccioli M. 1994. Rapid determination of phenol content in extra virgin olive oil. Grasas y Aceites 45, 68- 70.

Frega N, Bocci, F. 2001. L'analisi rápida dell' olio di oliva (3) (28). Laboratorio 2000, Italia.

García JM, Gutiérrez, Barrera MJ, Albi MA. 1996a. Storage of mill olives on an industrial scale. Journal of Agricultural and Food Chemistry 44, 590- 593.

García JM, Seller S, Pérez- Camino MC. 1996b. Influence of fruit ripening on olive oil quality. Journal of Agricultural and Food Chemistry 44, 3516- 3520.

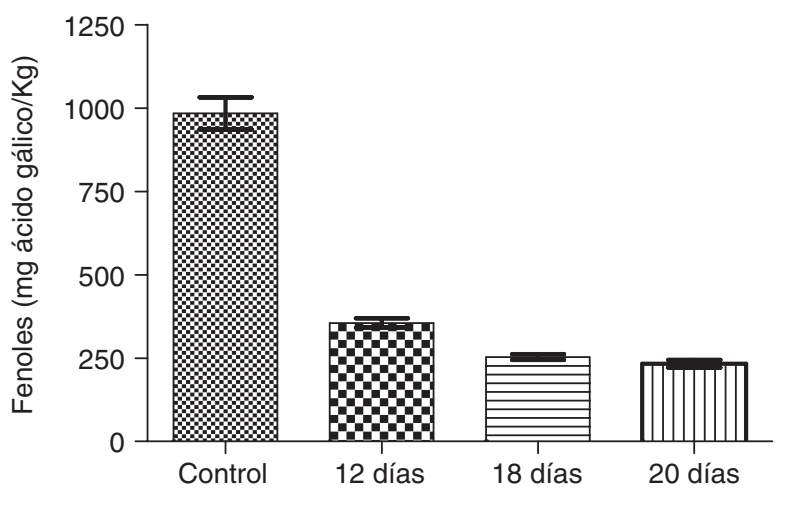

Figura 2

Contenido en fenoles totales ( $\mathrm{mg}$ ácido gálico/ $\mathrm{Kg}$ ) en aceite de oliva de la variedad Racimilla extraído tras la recolección (control) y tras 12, 18 y 20 días de almacenamiento (valor medio \pm desviación estándar, $\mathrm{n}=3$ ). 
García JM, Yousfi K. 2006. The postharvest of mill olives. Grasas y Aceites 57 (1) 16- 24.

Hermoso M, Uceda M, García A, Morales B, Frías M L y Fernández, A. 1991. Elaboración de aceite de oliva de calidad. Consejería de Agricultura y Pesca, Serie Apuntes 5/ 92. Sevilla. España.

Martínez- Suárez JM, Muñoz Aranda E, Alba Mendoza J y Lanzón Rey A. 1975. Informe sobre la utilización del Analizador de Rendimientos Abencor. Grasas y Aceites 26 (6) 379- 385.

Mateos R, Dominguez M M, Espartero JL, Cert A. 2003. Antioxidant effect of phenolic compounds alpha- tocopherol and other minor components in virgin olive oil. Journal of Agricultural and Food Chemistry 51, 71707175.

Mínguez- Mosquera M I, Rejano- Navarro L, Gandul- Rojas B, Sánchez- Gómez A H y Garrido- Fernández J. 1991. Color- pigment correlation in virgin olive oil. Journal of the American Oil Chemists Society 68 (5) 332- 336.

Montedoro G, Bertuccioli M, Anichini F. 1978. Aroma analisis of virgen olive oil by headspace (volatiles) and extraction (polyphenols) techniques. Flavour of Foods and Beverages 247- 281. Ed. G. Charalambous y G. E. Inglett. Nueva York: Academic Press.

Priego JM. 1930. Las variedades del olivo en Aragón y Rioja. Servicio de Publicaciones Agrícolas. Ministerio de Fomento. Dirección General de Agricultura y Montes.

Reglamento (CE) 2568/ 1991 de la Comisión relativo a las características de los aceites de oliva y de los aceites de orujo de oliva y sobre sus métodos de análisis.

Reglamento (CE) 1989/ 2003 de la Comisión que modifica el Reglamento (CE) 2568/ 1991 relativo a las características de los aceites de oliva y de los aceites de orujo de oliva y sobre sus métodos de análisis.

Reglamento (CE) 640/ 2008 de la Comisión que modifica el Reglamento (CE) 2568/ 1991 relativo a las características de los aceites de oliva y de los aceites de orujo de oliva y sobre sus métodos de análisis.

Salvador MD, Aranda F, Gómez- Alonso S, Fregapane G. 2001. Influence of fruit ripening on Cornicabra virgin olive oil quality. Food Chemistry 73, 45- 53

Toro MD, Sánchez MT, Montes F. 2002. La aceituna, calidad post- cosecha. Alimentación, Equipos y Tecnología 174, 75- 77.

Uceda M, Jiménez A, Beltrán G. 2006. Olive oil extraction and quality. Grasas y Aceites 57 (1) 25- 31. 